\title{
Stabilizing Controllers for Landmark Navigation of Planar Robots in an Obstacle-Ridden Workspace
}

\author{
Avinesh Prasad $\left(\mathbb{D}\right.$, Bibhya Sharma $\left(\mathbb{D}\right.$, Jito Vanualailai $\mathbb{D}^{D}$, and Sandeep Kumar $(\mathbb{D}$ \\ School of Computing, Information \& Mathematical Sciences, The University of the South Pacific, Suva, Fiji \\ Correspondence should be addressed to Bibhya Sharma; bibhya.sharma@usp.ac.fj
}

Received 1 June 2020; Revised 27 October 2020; Accepted 19 November 2020; Published 11 December 2020

Academic Editor: David Rey

Copyright (c) 2020 Avinesh Prasad et al. This is an open access article distributed under the Creative Commons Attribution License, which permits unrestricted use, distribution, and reproduction in any medium, provided the original work is properly cited.

\begin{abstract}
This paper essays a new solution to the landmark navigation problem of planar robots in the presence of randomly fixed obstacles through a new dynamic updating rule involving the orientation and steering angle parameters of a robot. The dynamic updating rule utilizes a first-order nonlinear ordinary differential equation for the changing of landmarks so that whenever a landmark is updated, the path followed by the robot remains continuous and smooth. This waypoints guidance is via specific landmarks selected from a new set of rules governing the robot's field of view. The governing control laws guarantee asymptotic stability of the $2 \mathrm{D}$ point robot system. As an application, the landmark motion planning and control of a car-like mobile robot navigating in the presence of fixed elliptic-shaped obstacles are considered. The proposed control laws take into account the geometrical constraints imposed on steering angle and guarantee eventual uniform stability of the car-like system. Computer simulations, using Matlab software, are presented to illustrate the effectiveness of the proposed technique and its stabilizing algorithm.
\end{abstract}

\section{Introduction}

Robots have a vast impact on our livelihood as they continue to improve efficiency, productivity, saving, quality, safety, security, and convenience of our endeavors, which are usually treated as being dull, dangerous, dirty, and difficult [1-3]. The applications of robots include search and rescue, surveillance, transportation, healthcare, pedestrian navigation, reconnaissance, pursuit-evasion, assembly, pick and place, and explorations in various environments [4-12]. Therefore, researchers around the world continuously and consistently come up with many robotic systems and a large array of real world applications and problems. Some of the prominent robotic systems addressed in literature include omniwheel robots $[6,13]$, car-like robots $[3,14,15]$, tractortrailer systems [16], mobile manipulators [2, 7], hexacopters [10], underwater robots [17], and Unmanned Aerial Vehicle (UAV) [18-20]. One problem which has received attention in the recent past is motion planning and control of robots, which is basically a coordinated and collision free movement, and completion of tasks either in known or unknown environments $[2,3,16,21,22]$. Within this motion planning and control problem, the recent influx and influence of the bioinspired behaviors from nature, which help in making inventions and designing algorithms and low-cost electromechanical tools for real life applications, have been noteworthy [23-25]. Some of the more common behaviors are formation, swarming, flocking, crawling, swimming, running, climbing, and motion camouflage [3, 24, 26, 27]. In addition, an assisting feature in nature to aid motion or movement is landmark. The landmarks are used by a number of insects and animals to navigate to a goal position in known or unknown environments [27-30].

A landmark is a geographic feature or visual cue which has been habitually used by insects and animals to guide them along their journey back home or to a foraging or nesting site $([27,28])$. Some examples from nature include the socially organized hymenoptera (ants, wasps, and honeybees), hamsters, and birds. According to Collett et al. [27], honeybees use landmarks to segment familiar routes. They can associate, with a landmark, a memory that encodes the direction and distance of the path segment between two consecutive landmarks. Etienne et al. [31] report that hamsters use external references to reset their path 
integrator by processing internal signals generated through rotations and translations. Harris et al. [32] state that wood ants utilize stored local view or snapshots of the landmarks situated close to their goals or destinations. The data are stored in a small set of distinct features, which greatly differs for the visually guided insects and birds. The landmarks have also been used by men in the early years and are also used in the current age of information and communications technologies (ICT). Explorers and sailors use natural and artificial landmarks to find their way back or through an area during their sea journey. Ships navigate within a veil of the lighthouse, which acts as an artificial landmark and helps prevent shipwrecking. More recently, navigation landmarks have been utilized for service robots in constrained environments [33], pedestrian autonomous navigation technology [12], long-range journeys through planetary explorations and rescue in hazardous environments [34], waypoints guidance $[13,17,35,36]$, and sampling of difficult environments.

It is noted that while natural landmarks are objects or features that are part of an environment and may have other functions not restricted to navigation, artificial landmarks are ones that need to be added to an environment for the sole purpose of robot navigation [29]. These landmarks can be incorporated with extra information, for example, in the form of RFID, bar codes, relevant sensors, and IR coding $[12,29,33]$. In literature, the concept of waypoints is linked to landmarks where the waypoints can be stipulated as the selected landmarks for a specific task or purpose. In addition, waypoints can also include physical objects, devices, or coordinates that hold navigation and location details which can be utilized to aid in robot navigation or human movement/following [13, 17].

The biologically inspired concept of landmarks has more recently been introduced in the field of robotics especially in the areas of path planning, communication, motion control, and navigation of autonomous robots, under biorobotics. The navigation problem can be either local navigation where the robot operates within a defined neighborhood such as the service robots in a home environment [33] or global navigation where the robot moves between different environments [30] such as the robots in outdoor applications (rescue in dangerous environments, pedestrian navigation [12]) and long-range journeys (planetary explorations [34]). Herein, the landmarks invariably serve two purposes: (1) to guide a robot to a desired goal [13,35-37] and (2) to enable a robot in determining its position with respect to the landmark (self-localization and mapping) [38-40]. Over the past two decades, majority of the researchers have primarily focussed on the detection of the landmarks and their selection based on user-defined metrics. The latter prominently depends on training and the new knowledge subsequently communicated to the rest of the robots.

The landmarks are detected using sensors; therefore, it is important that landmarks have distinct feature(s) distinguishing them from the other objects in the environment or workspace. Among many others in the literature, the works of Jagannathan [41], Lee [42], Fujii et al. [40], Ishii et al. [33], and Chand and Yuta [43] in this area are noteworthy. Jagannathan [41] used colors as distinguishing characteristic for landmarks so that the robot can differentiate and recognize these from other objects. Lee [42] and Ishii et al. [33] used infrared identification fused with encoder information, while Fujii et al. [40] used metallic landmarks to guide the mobile robots. Recently, Xie et al. in [19] presented a landmark detection and recognition algorithm for UAV Autonomous Pitching.

Once a set of landmarks in a definite workspace has been detected, it is imperative that a robot selects an optimal number and the exact landmarks which will be the waypoints to navigate along its way to target. Taking into account the cost and time constraints, it is impractical for a robot to navigate through all the landmarks in the workspace [44]. In the interest of brevity, selected work in this area which has considered different metrics for the selection of landmarks is presented. Deng et al. [45] in 1996 proposed a solution for the selection of landmarks from multiple landmarks so that the cost of sensing is minimized. Marsland et al. [46] in 2001 presented an automatic landmark selection algorithm that allowed a mobile robot to select conspicuous landmarks from a continuous stream of sensory perceptions, without any prior knowledge or human intervention during the selection process. Frommberger [44] in 2008 investigated a qualitative representation of landmarks for the selection process, while Beinhofer et al. [47] linearized the whole navigation cycle representing the landmark locations by a discrete set and then used a user-defined bound for conservative approximation of landmark visibility and selection. Lee et al. [48] presented a deep neural network-based landmark selection method for optical navigation on lunar highlands.

This paper focusses on the landmark navigation problem of planar robots and presents a solution based on a new dynamic updating rule tagged to a robot's orientation and steering angle. If a workspace contains few landmarks, then a $2 \mathrm{D}$ point robot can easily navigate to its target via all landmarks. However, a desired and optimized solution is needed to select a set of landmarks from a cohort of landmarks fixed randomly in a workspace. An algorithm will be designed to select only those landmarks that lie in the robot's field of view so that the robot can navigate to its goal via this selection, in the presence of obstacles.

This new scheme for selection and changing of landmarks, as well as the construction of the nonlinear control laws, is better than the other control schemes because of the following:

(1) For the selection of landmarks, the robot picks out only those landmarks that are intersecting with the field of view. These can be classified as waypoints guiding navigation. The updating rule tagged to the robot's orientation and steering angle allows for a smooth landmark switching process.

(2) The new method is systematic, elegant, and yet simple compared to, for example, the Lyapunovbased control scheme $[2,3,7]$, where there is no definite and standard procedure of constructing the total potentials. 
(3) The control laws ensure asymptotic stability of the $2 \mathrm{D}$ point robot system. With the introduction of the field of view, the Euclidean distance between the robot's position and its target is always strictly decreasing; hence, the Direct Method of Lyapunov is easily utilized to prove that the equilibrium point of the system governing the motion of the robot is asymptotically stable.

(4) The algorithm for $2 \mathrm{D}$ point robots can be easily applied to other planar robots such as car-like robots, tractor-trailer systems, and mobile manipulators. As an illustration, the navigation of the car-like robot is guided via waypoints or specific landmarks selected from the robot's field of view, guaranteeing eventual uniform stability of the system using theorem from Yoshizawa [49].

The main contributions of this research are as follows: (1) a new dynamic updating rule based on the orientation and steering angle of a robot for the selection of a set of relevant landmarks; (2) the velocity-based controllers which guarantee asymptotic stability of 2D point robot system; and (3) a new set of rules governing the robot's field of view for waypoints. As an application, the nonlinear controls of a nonholonomic car-like robot in the presence of obstacles are considered, validating the updating rule and eventually uniform stability of the robotic system.

The rest of the paper is organized as follows: In Section 2, the definition of workspace and point mass is given followed by the kinematic model of a $2 \mathrm{D}$ point robot. Section 3 gives a solution to the landmark navigation problem where the robot has to maneuver from an initial position to the target. In Section 4, obstacles are introduced in the workspace. Section 5 discusses the landmark navigation of a car-like mobile robot as an application. In Section 6, the strengths and weaknesses of the proposed method are discussed. Finally, Section 7 provides a discussion on the contributions and future research possibilities for the landmark navigation problem.

\section{Framework and Objective}

The nomenclature of $[7,50]$ is adopted to define and set the parameters of the research framework.

Definition 1. The workspace is a fixed, closed, and bounded rectangular region for some $\eta_{1}>0$ and $\eta_{2}>0$. Precisely, the workspace is the set WS $=\left\{\left(z_{1}, z_{2}\right) \in \mathbb{R}^{2}: 0 \leq z_{1} \leq \eta_{1}, 0\right.$ $\left.\leq z_{2} \leq \eta_{2}\right\}$.

Definition 2. Let $P$ be a $2 \mathrm{D}$ point robot in the $z_{1} z_{2}$ plane, positioned at $(x, y)$ with a circular protective region of radius $r_{P} \geq 0$ and moving with a velocity of $v$ at time $t \geq 0$. The $2 \mathrm{D}$ point is a set

$$
P=\left\{\left(z_{1}, z_{2}\right) \in \mathbb{R}^{2}:\left(z_{1}-x\right)^{2}+\left(z_{2}-y\right)^{2} \leq r_{P}^{2}\right\} .
$$

Now, suppose that $u_{1}$ and $u_{2}$ are the $z_{1}$ and $z_{2}$ components, respectively, of $v$; then, the kinematic model of $P$ can be expressed as

$$
\left.\begin{array}{ll}
\dot{x}=u_{1}, & \dot{y}=u_{2}, \\
x_{0}:=x\left(t_{0}\right), & y_{0}:=y\left(t_{0}\right) .
\end{array}\right\}
$$

System (1) is a description of the instantaneous velocities of the $2 \mathrm{D}$ point robot, where $u_{1}$ and $u_{2}$ are classified as the velocity-based controllers. Hereafter, the vector notation $\mathbf{x}=$ $(x(t), y(t))$ is used to refer to the position of the 2D point robot in the $z_{1} z_{2}$-plane.

Sharma et al. in $[2,3,7]$ designed continuous nonlinear controllers $u_{1}$ and $u_{2}$ using a new Lyapunov-based control scheme (LbCS) so that the robot moves straight from an initial to the goal position. In this paper, the same motion planning and control (MPC) problem is solved, but the robot will be guided to its goal by selected landmarks in the workspace, essentially the landmark navigation problem. The problem statement is as follows.

Given a set of landmarks in the $z_{1} z_{2}$-plane, design the controllers $u_{1}$ and $u_{2}$ so that the robot can navigate using landmarks based upon some a priori known metric and reach the target. If the workspace contains multiple landmarks scattered randomly, then design an algorithm which can select a subset of those landmarks that can be used to guide the robot to its target. Moreover, if the workspace is cluttered with circular or elliptical obstacles, then the robot should be able to successfully avoid them and reach the target safely via the landmarks.

The control scheme showing the design of the controllers is shown in Figure 1. The scheme will consider all the obstacles in the workspace, thus giving the motion planner a global view of the workspace and picking out the safest path among the obstacles. The turning/steering angle of the robot as a function of the reciprocal of the distance from the robot to an obstacle is designed. Since this distance appears in the denominator, the magnitude of turning/steering angle will increase as the robot approaches an obstacle, thus deviating the robot away from the obstacle.

\section{A Solution to the Landmark Navigation Problem}

Definition 3. The $k$ th landmark with the position (2D Euclidean) coordinates $\left(l x_{k}, l y_{k}\right)$ in the $z_{1} z_{2}$-plane is given by

$$
\operatorname{LM}_{k}=\left\{\left(z_{1}, z_{2}\right) \in \mathbb{R}^{2}:\left(z_{1}-l x_{k}\right)^{2}+\left(z_{2}-l y_{k}\right)^{2}=0\right\},
$$

for $k=1,2, \ldots, r$.

Assumption 1. The positions of the landmarks are a priori known.

Assumption 2. The robot goes through each of the landmarks selected by the dynamic updating rule.

Consider a scenario where the $2 \mathrm{D}$ point robot has to maneuver via landmarks randomly fixed in WS en route to its target. To solve this problem, let $d_{k}(t)=\|\left(x-l x_{k}, y-\right.$ $\left.l y_{k}\right) \|$ for $k=1,2, \ldots, r$ be the distance between the 2D point robot, $P$, and the $k$ th landmark, $\mathrm{LM}_{k}$, at time $t \geq 0$ and define $\left(l x_{r+1}, l y_{r+1}\right):=\left(p_{1}, p_{2}\right)$. Assume that $d_{1}<d_{2}<\cdots<d_{r}$ and a velocity of 


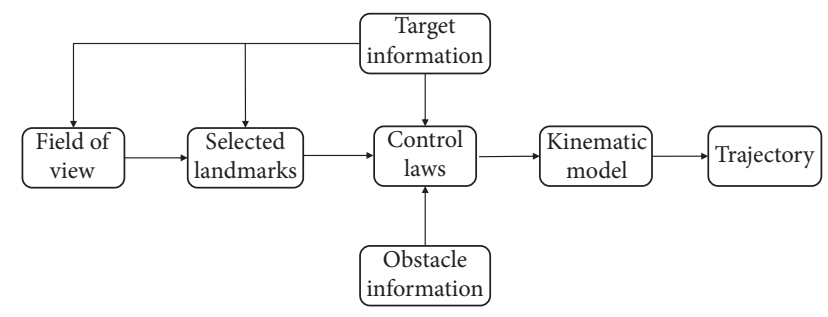

Figure 1: Block diagram illustrating the control scheme.

$$
v(t)=\mu\left\|\left(x-p_{1}, y-p_{2}\right)\right\|,
$$

(where $\mu>0$ is a constant) is applied to the 2D point robot $P$ in the direction $\psi(t)$ measured from the positive $z_{1}$-axis. Then the controllers $u_{1}$ and $u_{2}$ can be expressed as

$$
\begin{aligned}
& u_{1}=v \cos \psi, \\
& u_{2}=v \sin \psi
\end{aligned}
$$

While the velocity algorithm given by (4) is capable of moving the robot, it is $\psi(t)$ that determines the direction of the robot's movement. Thus, the landmark navigation problem can be solved by carefully defining $\psi(t)$. Two simple cases are illustrated below.

Case 1. Navigation via all landmarks

For $P$ to navigate via all the landmarks, the following differential form of $\psi(t)$ is proposed:

$$
\begin{aligned}
& \dot{\psi}(t)=\left\{\begin{array}{l}
0, \quad \text { if } d_{k} \neq 0 \text { or }(x(t), y(t))=\left(p_{1}, p_{2}\right), \\
\tan ^{-1}\left[\left(l y_{k+1}-y(t)\right) \cos \psi(t)-\left(l x_{k+1}-x(t)\right) \sin \psi(t)\right], \quad \text { if } d_{k}=0,
\end{array}\right. \\
& \psi(0)=a \tan 2\left[l y_{1}-y(0), l x_{1}-x(0)\right],
\end{aligned}
$$

for $t>0$ and $k=1,2, \ldots, r$.

The differential form governed by (4) will ensure that the robot will navigate through all the landmarks in the definite and bounded workspace en route to its target. While the workspace can contain landmarks scattered all over, it is impractical, costly, laborious, and with no real need for the robot to navigate using all the landmarks [44]. The following case defines a new procedure of selecting only the landmarks which fall under a new set of metrics.

Case 2. Navigation via selected landmarks

Assume that the workspace contains finitely many landmarks scattered all over. Only those landmarks that fall under a new set of metrics are selected, which essentially defines a new field of view of the robot between its initial and target positions. A unique field of view is defined and introduced in this paper as follows.

Definition 4. Given a predetermined scalar $\zeta \in(0,(\pi / 2))$, the set

$$
\begin{aligned}
\mathrm{FV} & =\left\{\left(z_{1}, z_{2}\right) \in \mathbb{R}^{2}: z_{1} \in x(t)+\lambda\left(p_{1}-x(t)\right) \cos \tau\right. \\
& -\lambda\left(p_{2}-y(t)\right) \sin \tau, z_{2} \in y(t)+\lambda\left(p_{2}-y(t)\right) \cos \tau \\
& \left.+\lambda\left(p_{1}-x(t)\right) \sin \tau, \quad 0 \leq \lambda \leq 1,|\tau| \leq \zeta\right\}
\end{aligned}
$$

is the robot's field of view. The newly stipulated field of view (FV) contains all the points in the $z_{1} z_{2}$ plane that lie within an angle of $\zeta$ on either side of the line joining the robot's current position and the target. This FV is illustrated in Figure 2 .

Note that the field of view is initially calculated at $t=0$ and is updated at each landmark. This would ensure that the robot does not unnecessarily change the field of view if it encounters obstacles on its way.
A metric for the selection of the landmarks is defined as follows:

(1) The selected landmark should lie within the FV and between the robot's position $(x, y)$ and its target $\left(p_{1}, p_{2}\right)$.

(2) The selected landmark should be at a sufficient distance $D^{*}>0$ away from the previous landmark. This would mean that no two selected landmarks are very close to each other (within a distance of $D^{*}>0$ ).

(3) The selected landmark should be at a sufficient distance $D^{*}>0$ away from the target.

(4) The next landmark should be selected only when the robot reaches its current landmark.

An algorithm for the selection of landmark based on the above metric is developed. This is described in Algorithm 1.

Algorithm 1. Let the selected landmark be denoted as $\left(l x^{*}, l y^{*}\right)$. Note that $\left(l x^{*}, l y^{*}\right)$ will be determined initially at $t=0$ and then updated when $\left\|\left(x(t)-l x^{*}, y(t)-l y^{*}\right)\right\|=0$ as it is desired that the robot actually goes through the selected landmark. Suppose that there are $r$ landmarks in the workspace. Let $S=\{1,2,3, \ldots, r\}$ such that $d_{1}<d_{2}<\cdots<d_{r}$. Next, the $k$ th subset, $S_{k} \subseteq S$, is defined as

$$
S_{k}= \begin{cases}\{k\}, & \text { if }\left(l x_{k}, l y_{k}\right) \in \mathrm{FV}, d_{k}>D^{*}, \\ & \left\|\left(l x_{k}-p_{1}, l y_{k}-p_{2}\right)\right\|>D^{*}, \\ \{\}, & \text { otherwise, }\end{cases}
$$

for $k=1,2, \ldots, r$. Then, the landmark to be selected is given by

$$
\left(l x^{*}, l y^{*}\right)= \begin{cases}\left(p_{1}, p_{2}\right), & \text { if } M=\{\} ; \\ \left(l x_{\min M}, l y_{\min M}\right), & \text { otherwise, }\end{cases}
$$




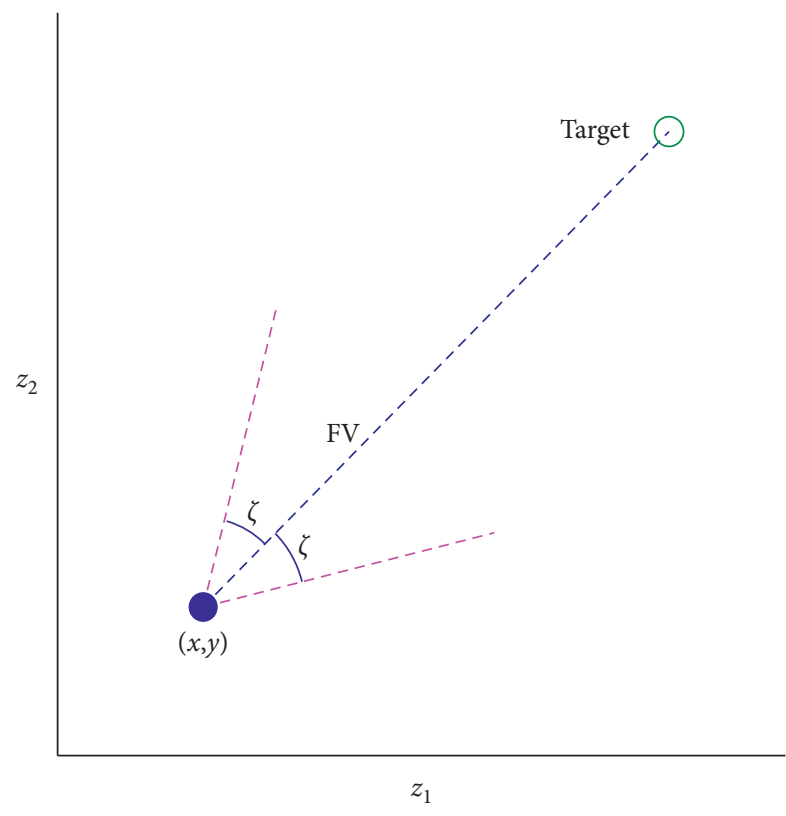

FIGURE 2: Schematic representation of the robot's field of view (FV).

where $M=\cup_{k=1}^{r+1} S_{k}$.

To ensure that the robot goes through the selected landmark, the following differential form of $\psi(t)$ is proposed:

$$
\begin{aligned}
& \dot{\psi}(t)=\tan ^{-1}\left[\left(l y^{*}-y(t)\right) \cos \psi(t)-\left(l x^{*}-x(t)\right) \sin \psi(t)\right], \\
& \psi(0)=a \tan 2\left[l y^{*}-y(0), l x^{*}-x(0)\right] .
\end{aligned}
$$

The effectiveness of the proposed solution is illustrated in Simulation 1.

Simulation 1. Figures 3 and 4 show simulations with different initial and final positions. The positions of the landmarks were randomly generated within the workspace. The initial and final positions are given in the figure captions while the constant $\zeta$ and $D^{*}$ are $\pi / 4$ and 5, respectively. As shown in the figures, with the landmarks scattered all over the workspace, the robot only navigates through selected landmarks which lie in its field of view. It can clearly be noticed that no two selected landmarks (or a selected landmark and the target) are within a distance of $D^{*}=5$ units.

The discussion so far can be summarized in the following theorem.

Theorem 1. Let $u_{1}$ and $u_{2}$ be as defined by (5). Then, the point $\mathbf{e}=\left(p_{1}, p_{2}\right)$ is the only equilibrium point of system (2) and is asymptotically stable.

Proof. Note that $(\dot{x}, \dot{y})=(0,0)$ only if $u_{1}=u_{2}=0$, which implies that $v(t)=0$. From (4), it is clear that $v(t)=0$ implies that $\mathbf{x}(t)=\left(p_{1}, p_{2}\right)$. Thus, $\mathbf{e}$ is the only equilibrium point of system (2).

To prove asymptotic stability, consider a Lyapunov function of the form

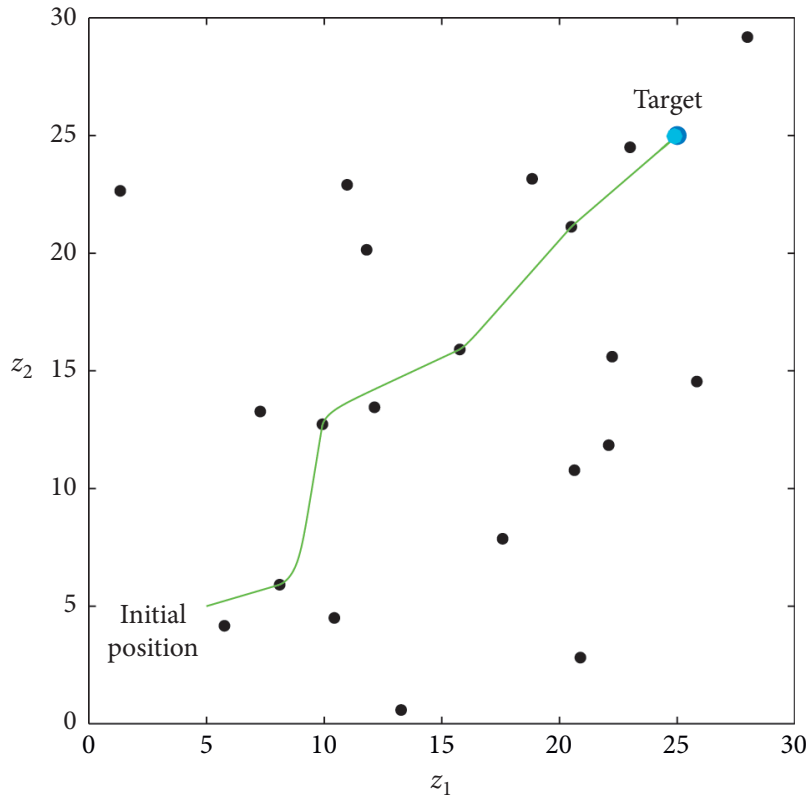

FIGURE 3: Landmark navigation of the $2 \mathrm{D}$ point robot with initial position $(5,5)$ and the target placed at $(25,25)$.

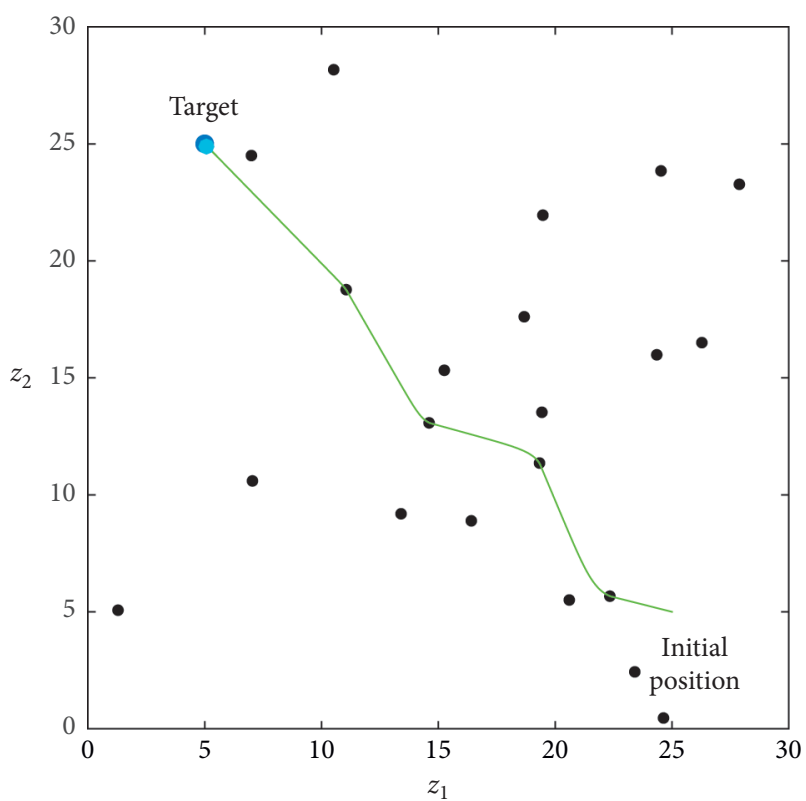

FIgURE 4: Landmark navigation of the $2 \mathrm{D}$ point robot with initial position $(25,5)$ and the target placed at $(5,25)$.

$$
L(\mathbf{x})=\frac{1}{2}\|\mathbf{x}(t)-\mathbf{e}\|^{2},
$$

which is defined, continuous, positive, and radially unbounded over the domain $D=\left\{\mathbf{x} \in \mathbb{R}^{2}\right\}$. Clearly, $L(\mathbf{x})$ has continuous first partial derivatives on the region $D$ of the neighborhood of the equilibrium point $\mathbf{e}$ of system (2). Moreover, in the region $D, L(\mathbf{e})=0$ and $L(\mathbf{x})>0$ for all $\mathbf{x} \neq \mathbf{e}$. Now, the time derivative of $L(\mathbf{x})$ along a trajectory of system (2) is given by 


$$
\dot{L}(\mathbf{x})=\left(x-p_{1}\right) u_{1}+\left(y-p_{2}\right) u_{2} .
$$

It is clear that, in the region $D, \dot{L}(\mathbf{e})=0$. Next, it will be shown that $\dot{L}(\mathbf{x})<0$ for all $\mathbf{x} \neq \mathbf{e}$. Note that, for $\mathbf{x} \neq \mathbf{e}$, the $\dot{L}(\mathbf{x})$ is simplified as

$$
\begin{aligned}
\dot{L}(\mathbf{x}) & =-v\left[\left(p_{1}-x\right) \cos \psi+\left(p_{2}-y\right) \sin \psi\right] \\
& =-\frac{v\left[\left(p_{1}-x\right)\left(l x^{*}-x\right)+\left(p_{2}-y\right)\left(l y^{*}-y\right)\right]}{\left\|\left(l x^{*}-x, l y^{*}-y\right)\right\|} .
\end{aligned}
$$

Note that $\left\|\left(l x^{*}-x, l y^{*}-y\right)\right\|>0$ since $\left(l x^{*}, l y^{*}\right)$ is updated as $\left\|\left(l x^{*}-x, l y^{*}-y\right)\right\| \longrightarrow 0$. Next, since $\zeta \in(0,(\pi / 2))$ and $\left(l x^{*}, l y^{*}\right) \in \mathrm{FV}$, it follows that the angle, $\beta$, between the nonzero vectors $\left(\begin{array}{l}p_{1}-x \\ p_{2}-y\end{array}\right)$ and $\left(\begin{array}{l}l x^{*}-x \\ l y^{*}-y\end{array}\right)$ satisfies $\beta \in(-\zeta, \zeta) \subseteq(-(\pi / 2),(\pi / 2))$. Thus,

$$
\begin{array}{r}
\left(p_{1}-x\right)\left(l x^{*}-x\right)+\left(p_{2}-y\right)\left(l y^{*}-y\right) \\
=\left(\begin{array}{l}
p_{1}-x \\
p_{2}-y
\end{array}\right) \cdot\left(\begin{array}{l}
l x^{*}-x \\
l y^{*}-y
\end{array}\right)>0 .
\end{array}
$$

In addition, since $v>0$, it is clear that $\dot{L}(\mathbf{x})<0$ for all $\mathbf{x} \neq \mathbf{e}$. Hence, it can be concluded that $\mathbf{e}$ is an asymptotically stable equilibrium point of system (2).

To numerically verify the stabilizing results obtained from the Lyapunov function, the graph of the Lyapunov function and its derivative for the trajectory shown in Figure 4 is generated. The graph of $L(\mathbf{x})$ and its time derivative $\dot{L}(\mathbf{x})$ is shown in Figure 5. One can notice that $L(\mathbf{x})$ is a monotonically decreasing and both $L(\mathbf{x})$ and $\dot{L}(\mathbf{x})$ vanish at the target.

\section{Inclusion of Obstacles in the Workspace}

The landmark navigating problem becomes even more challenging when the WS is injected with stationary obstacles. Researchers have used various schemes and strategies for avoiding obstacles of various kinds (artificial, moving, static) and shapes. Some of the prominent ones in the literature are potential field method [2, 3], artificial neural networks [22], and geometric based approach [51], to name a few. In addition, the authors of this research have designed an approach similar to the one discussed in the paper for obstacle and collision avoidance alike. The reader is referred to [51] for a detailed account of the avoidance scheme.

Let $q>0$ solid bodies be fixed within WS. Elliptic obstacles of random sizes are considered since most 2-dimensional objects can be represented in an elliptic form or can be inscribed within an ellipse with minimum overlap.

Definition 5. The $l$ th elliptic obstacle with center $\left(o_{l 1}, o_{l 2}\right)$ and constants $a_{l}>0$ and $b_{l}>0$ on the $z_{1} z_{2}$ plane is described as

$$
\mathrm{FO}_{l}=\left\{\left(z_{1}, z_{2}\right) \in \mathbb{R}^{2}: \frac{\left(z_{1}-o_{l 1}\right)^{2}}{a_{l}^{2}}+\frac{\left(z_{2}-o_{l 2}\right)^{2}}{b_{l}^{2}} \leq 1\right\},
$$

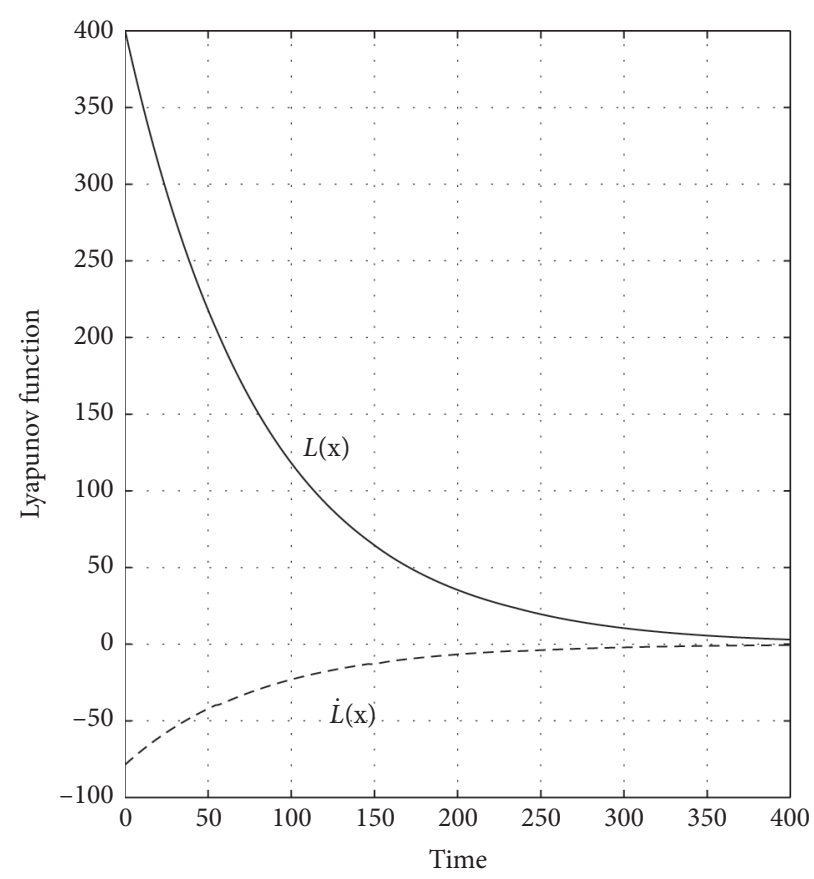

FIgURE 5: Evolution of the Lyapunov function (solid line) and its derivative (dashed line) for the trajectory shown in Figure 4.

for $l=1,2, \ldots, q$.

Definition 6. The region surrounding the elliptic-shaped obstacles given by the set

$$
S=\bigcup_{l=1}^{q}\left\{\left(z_{1}, z_{2}\right) \in \mathbb{R}^{2}: \frac{\left(z_{1}-o_{l 1}\right)^{2}}{\left(a_{l}+d_{\max }\right)^{2}}+\frac{\left(z_{2}-o_{l 2}\right)^{2}}{\left(b_{l}+d_{\max }\right)^{2}} \leq 1\right\},
$$

is denoted as the sensing zone, where $d_{\max }>0$ is a control parameter used to determine the size of the sensing zone.

Assumption 3. The selected landmark does not intersect with the sensing zone.

For the 2D point robot $P$ to avoid the stationary obstacles while navigating via the landmarks, the idea proposed by Prasad et al. in $[14,51]$ is adopted. The controllers $u_{1}$ and $u_{2}$ are defined as

$$
\begin{aligned}
& u_{1}=v \cos (\psi+\varepsilon), \\
& u_{2}=v \sin (\psi+\varepsilon),
\end{aligned}
$$

where $\varepsilon$ determines the direction in which the 2D point robot $P$ should turn to avoid an obstacle on its path, while the differential form of $\psi(t)$ is given in (10).

Figure 6 shows the proposed path robot $P$ should follow to avoid an obstacle along its path to the $k$ th landmark. Let $R_{l}=$ $\left(\left(x-o_{l 1}\right) / a_{l}\right)^{2}+\left(\left(y-o_{l 2}\right) / b_{l}\right)^{2}-1$ be a measure of the distance from $P$ to the circumference of the $l$ th fixed obstacle; then,

$$
\varepsilon=\tan ^{-1}\left(\sum_{l=1}^{q} \frac{\alpha_{l} \beta_{l}}{R_{l}}\right)
$$




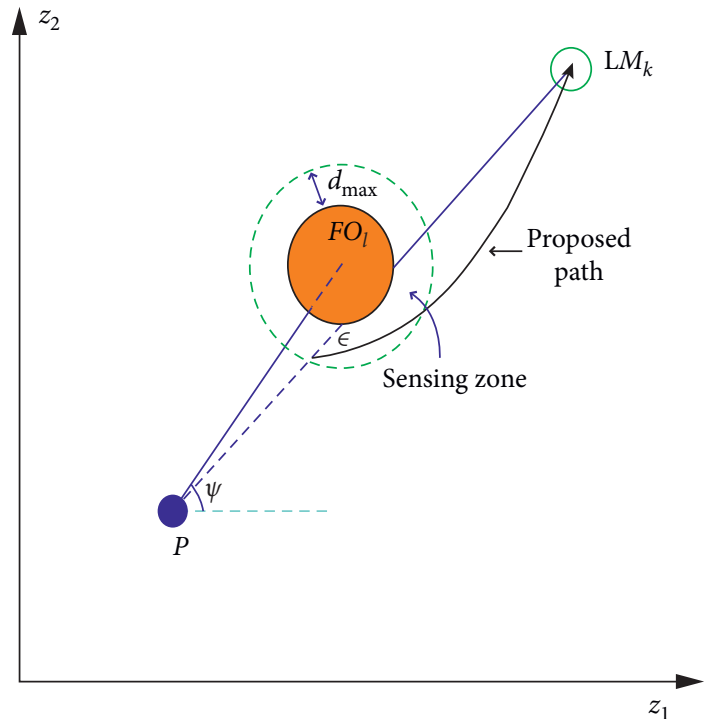

FIgURE 6: Schematic representation of the avoidance scheme with parameter $d_{\text {max }}$ (modified from [51]).

where $\alpha_{l}=\max \left\{0, d_{\max }-R_{l}\right\}$ and

$$
\beta_{l}= \begin{cases}1, & \text { if }\left(x-o_{l 1}\right)\left(l y^{*}-y\right)-\left(y-o_{l 2}\right)\left(l x^{*}-x\right) \leq 0, \\ -1, & \text { if }\left(x-o_{l 1}\right)\left(l y^{*}-y\right)-\left(y-o_{l 2}\right)\left(l x^{*}-x\right)>0 .\end{cases}
$$

Substitute (18) into (17) and simplify to get

$$
\begin{aligned}
{\left[\begin{array}{l}
u_{1} \\
u_{2}
\end{array}\right]=} & \frac{\mu\left\|\left(x-p_{1}, y-p_{2}\right)\right\|}{\left\|\left(1, \sum_{l=1}^{q} \alpha_{l} \beta_{l} / R_{l}\right)\right\|} \\
\times & {\left[\begin{array}{cc}
1 & -\sum_{l=1}^{q} \frac{\alpha_{l} \beta_{l}}{R_{l}} \\
\sum_{l=1}^{q} \frac{\alpha_{l} \beta_{l}}{R_{l}} & 1
\end{array}\right]\left[\begin{array}{c}
\cos \psi \\
\sin \psi
\end{array}\right], }
\end{aligned}
$$

where the function $\psi(t)$ is as defined in (10). Since the selected landmark should not intersect with the sensing zone, the set $S_{k}$ in Algorithm 1 is refined as

$$
S_{k}= \begin{cases}\{k\}, & \text { if }\left(l x_{k}, l y_{k}\right) \in \mathrm{FV},\left(l x_{k}, l y_{k}\right) \notin S, \\ d_{k}>D^{*}, & \left\|\left(l x_{k}-p_{1}, l y_{k}-p_{2}\right)\right\|>D^{*} \\ \{\}, & \text { otherwise, }\end{cases}
$$

for $k=1,2, \ldots, r$.

Simulation 2. Figures 7 and 8 show simulations with different sets of initial and final positions of robot $P$. With the landmarks and elliptic-shaped obstacles (with randomized radii) randomly scattered over the workspace, the robot only navigates through selected landmarks which lie in its field of view while avoiding any obstacle that it encounters along its path. The circular objects (in red) are the stationary obstacles while the

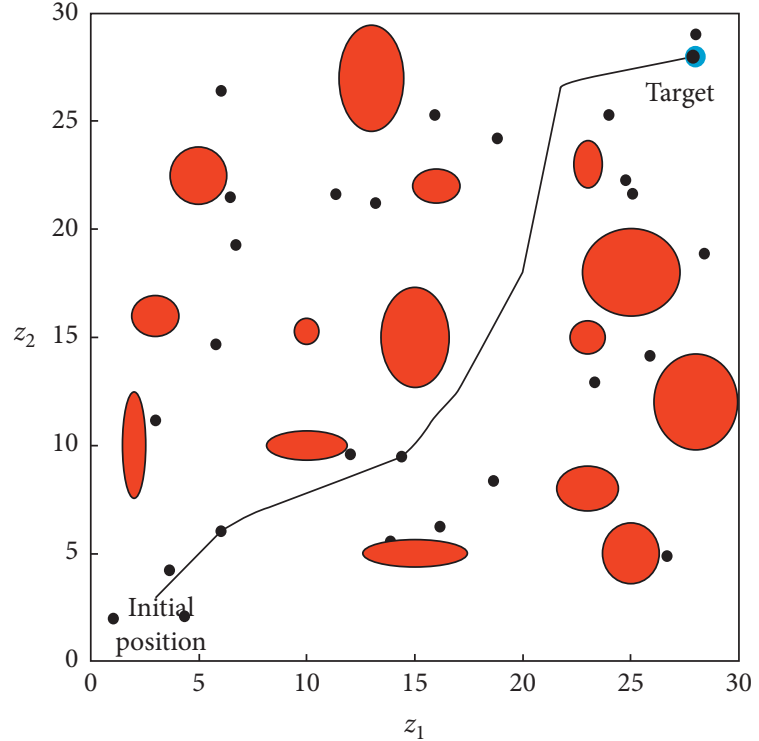

FIGURE 7: Landmark navigation of the 2D point robot with initial position $(3,3)$ and the target placed at $(28,28)$.

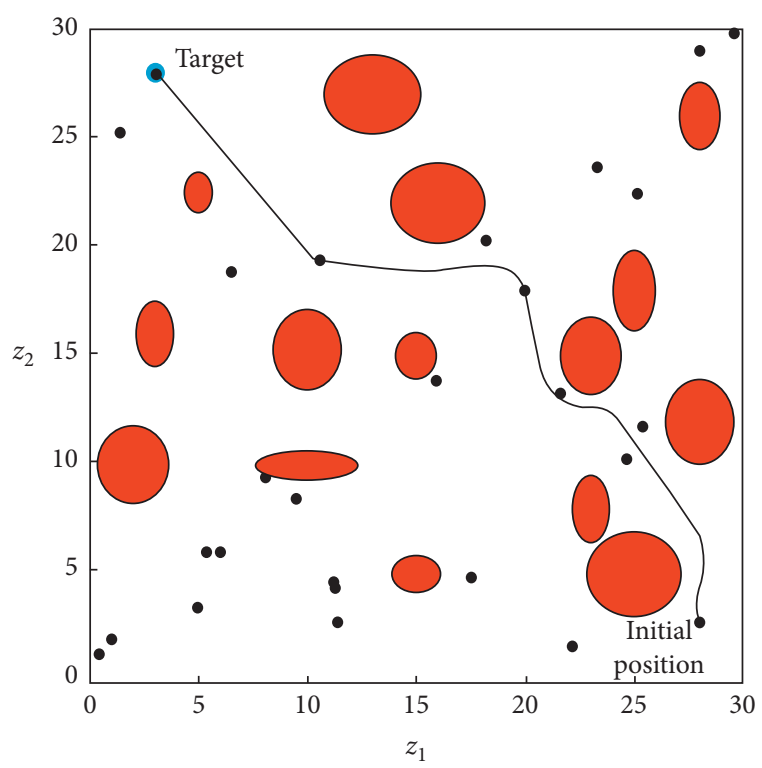

FIgURE 8: Landmark navigation of the $2 \mathrm{D}$ point robot with initial position $(28,3)$ and the target placed at $(3,28)$.

black dots represent the landmarks. Constant $\zeta=\pi / 6, D^{*}=3$, and $d_{\max }=3$ for both simulations. With regard to the evolution of $L(\mathbf{x})$ and $\dot{L}(\mathbf{x})$, a similar trend to those of the previous example is seen, again reaffirming that $L(\mathbf{x})$ is a decreasing function and both $L(\mathbf{x})$ and $\dot{L}(\mathbf{x})$ vanish at the target.

\section{Application: Landmark Navigation of a Car-like Robot}

In this section, the technique proposed in the above sections is applied to address the landmark navigation problem of a car-like mobile robot containing nonholonomic constraints. 


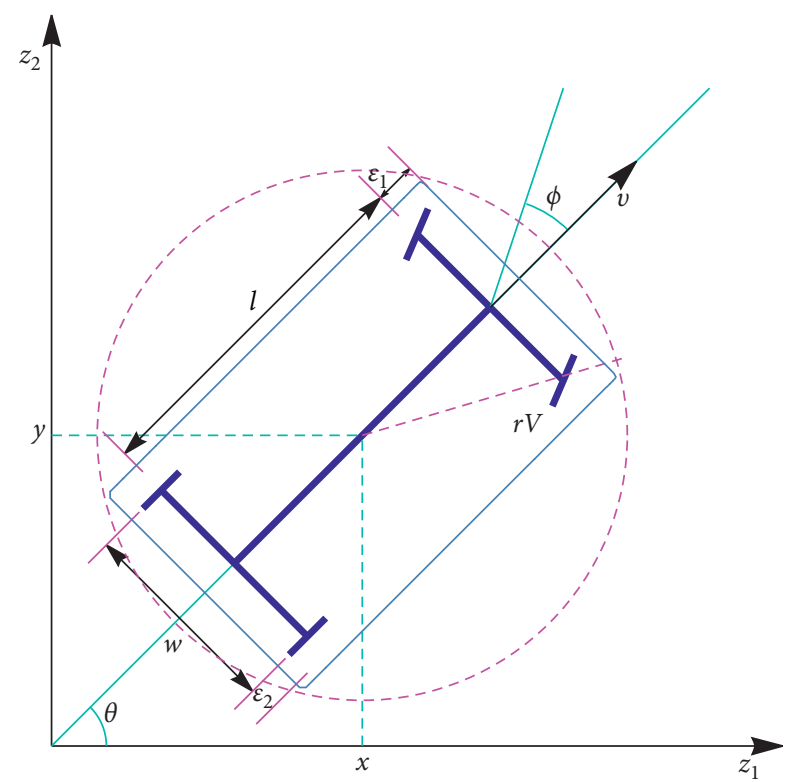

Figure 9: A rear wheel driven vehicle with front wheel steering and steering angle $\phi$ adopted from [14].

The rear wheel driven car-like vehicle model is adopted from [7]. Referring to Figure 9, $(x, y)$ denotes the center of mass (CoM) of the car, $\theta$ gives the car's orientation with respect to the $z_{1}$-axis, and $\phi$ gives the steering wheel's angle with respect to car's longitudinal axis. The constants $\varepsilon_{1}$ and $\varepsilon_{2}$ are the clearance parameters. The configuration of the car is given by $(x, y, \theta, \phi) \in \mathbb{R}^{4}$, and its position is given as the point $(x, y) \in \mathbb{R}^{2}$.

The configuration of the car is given by $(x, y, \theta, \phi) \in \mathbb{R}^{4}$, and its position is given as the point $\mathbf{x}^{*}=(x, y) \in \mathbb{R}^{2}$. If $\ell$ is the distance between the two axles and $w$ the length of each axle, then the kinematic model of the car-like vehicle is given by

$$
\left.\begin{array}{l}
\dot{x}=v \cos \theta-\frac{v}{2} \tan \phi \sin \theta, \\
\dot{y}=v \sin \theta+\frac{v}{2} \tan \phi \cos \theta, \\
\dot{\theta}=\frac{v}{\ell} \tan \phi,
\end{array}\right\}
$$

where $v$ is the translational velocity and $\phi$ is the steering angle.

Hereafter, the vector notation $\mathbf{x}=(x, y, \theta) \in \mathbb{R}^{3}$ shall be used to describe the variables in (22), while $v$ and $\phi$ are treated as controllers. In addition, no slippage (i.e., $\dot{x} \sin \theta-\dot{y} \cos \theta=0$ ) and pure rolling (i.e., $\dot{x} \cos \theta+\dot{y} \sin \theta=v$ ) of the platform wheels are assumed. These nonintegrable constraints, denoted as the nonholonomic constraints, are captured in system (22).

Assume that there are $q \in \mathbb{N}$ stationary solid objects which the car-like robot has to avoid while navigating using the landmarks to reach its target $\left(p_{1}, p_{2}\right)$. The stationary objects are defined in Definition 5 and the sensing zone is provided in Definition 6. Assume that the target position does not intersect with the sensing zone. For the robot to steer safely past an obstacle, the entire vehicle is enclosed in the smallest circle possible with center $(x, y)$ and radius

$$
r_{V}=\frac{1}{2}\left\|\left(\ell+2 \varepsilon_{1}, w+2 \varepsilon_{2}\right)\right\|,
$$

as shown in Figure 9. Since a nonholonomic vehicle is considered, it is important for safety of the vehicle that the robot must slow down on approach to an obstacle $[7,14,51]$. Moreover, the vehicle's steering angle is restricted as $|\phi(t)| \leq \phi_{\max }$, where $\phi_{\max }<\pi / 2$ is the maximum steering angle $[2,7]$. A limitation on the steering angle is important for the safety of the car-like robot as well as for preventing situations where the robot can get jammed when its front wheel is normal to the longitudinal axis of its body. Next, assume that there are $r$ landmarks scattered randomly in WS. For the selection of the landmarks that lie in the robot's FV, Algorithm 1 developed in Section 3 is used. For avoidance of the solid objects and convergence to the target via landmarks, the following forms of the controllers $v(t)$ and $\phi(t)$ are proposed:

$$
\left\{\begin{array}{l}
v(t)=\mu\left\|\left(x(t)-p_{1}, y(t)-p_{2}\right)\right\| \prod_{l=1}^{q}\left(1-\frac{\alpha_{l}(t)}{d_{\max }}\right), \\
\phi(t)=\frac{2 \phi_{\text {max }}}{\pi} \tan ^{-1}\left(\xi(t)+\sum_{l=1}^{q} \frac{\alpha_{l}(t) \beta_{l}(t)}{R_{l}(t)}\right),
\end{array}\right.
$$

where $\xi(t)$ is obtained by the numerically solving the differential equation

$$
\begin{aligned}
& \dot{\xi}(t)=\frac{\left(l y^{*}-y(t)\right) \cos \theta(t)-\left(l x^{*}-x(t)\right) \sin \theta(t)}{\left\|\left(l x^{*}-x(t), l y^{*}-y(t)\right)\right\|+\gamma}-\xi(t), \\
& \xi(0)=a \tan 2\left(l y^{*}-y(0), l x^{*}-x(0)\right)-\theta(0) .
\end{aligned}
$$

The constants $\mu>0$ and $\gamma>0$ are user-defined, and the variables $R_{l}(t), \alpha_{l}(t)$, and $\beta_{l}(t)$ are

$$
\begin{aligned}
& R_{l}(t)=\left\|\left(\frac{x(t)-o_{l 1}}{a_{l}+r_{V}}, \frac{y(t)-o_{l 2}}{b_{l}+r_{V}}\right)\right\|^{2}-1, \\
& \alpha_{l}(t)=\max \left\{0, d_{\max }-R_{l}(t)\right\}, \\
& \beta_{l}(t)= \begin{cases}1, & \text { if }\left(x(t)-o_{l 1}\right)\left(l y^{*}-y(t)\right) \\
-1, & \text { if }\left(x(t)-o_{l 1}\right)\left(l y^{*}-y(t)\right) \\
- & -\left(y(t)-o_{l 2}\right)\left(l x^{*}-x(t)\right)>0 .\end{cases}
\end{aligned}
$$


The controller $\phi(t)$ is bounded as $-\phi_{\max }<\phi(t)<\phi_{\max }$ for all $t>0$. This is because $\tan ^{-1}(\cdot)$ is bounded as $\left|\tan ^{-1}(\cdot)\right|<(\pi / 2)$. Hence,

$$
|\phi(t)|<\frac{2 \phi_{\max }}{\pi} \cdot \frac{\pi}{2}=\phi_{\max }, \quad \forall t>0,
$$

or equivalently $-\phi_{\max }<\phi(t)<\phi_{\max }$ for all $t>0$. Also note that the term $\prod_{l=1}^{q}\left(1-\left(\left(\alpha_{l}(t)\right) / d_{\max }\right)\right)$ will ensure that the robot slows down as it approaches an obstacle. The landmark position $\left(l x^{*}, l y^{*}\right)$ is determined using Algorithm 1, except that the set $S_{k}$ is defined as

$$
S_{k}=\left\{\begin{array}{l}
\{k\}, \quad \text { if }\left(l x_{k}, l y_{k}\right) \in \mathrm{FV},\left(l x_{k}, l y_{k}\right) \notin S, \\
\quad d_{k}>D^{*},\left\|\left(l x_{k}-p_{1}, l y_{k}-p_{2}\right)\right\|>D^{*} \\
\{\}, \quad \text { otherwise, }
\end{array}\right.
$$

for $k=1,2, \ldots, r$.

Simulation 3. Figures 10 and 11 show simulations with different initial and final positions of the car-like robot. With the landmarks and obstacles (random size) scattered randomly all over the workspace, the robot navigates through landmarks which lie in its FV while avoiding any fixed obstacles that it encounters along its path to the target. Table 1 gives the values of the parameters used in the simulations.

Figures 12 and 13 show the evolution of controllers for the trajectory shown in Figures 10 and 11, respectively. The controllers are continuous for all time $t>0$ and vanish as the robot reaches its target. The concave sections of the velocity graph indicate that the robot slowed down on approach to obstacles. After it avoids collision with the fixed obstacles, the robot gained speed but then finally slowed down on approach to the target. Eventually, at the center of the target, the velocity and the steering angle became zero.

5.1. Stability of System (22). As seen in the simulations for the car-like robot, the planar trajectory $\mathbf{x}^{*}(t)=\left(x_{0}(t), y_{0}(t)\right)$ of system (22) is, in general, eventually stable, which means that initially the system trajectory could move away from the target $\mathbf{p}=\left(p_{1}, p_{2}\right)$, but after a certain time $T>0$ the trajectory converges to a vicinity of the target and remain there for all time $t \geq T$.

The concept of eventual stability was developed by Yoshizawa [49] in 1966. This is briefly described below and the reader can refer to [16] for more details.

Let $I$ denote the interval $0 \leq t<\infty$ and $\mathbb{R}^{n}$ denote Euclidean $n$-space. Consider a system of differential equations

$$
\dot{X}=F(t, X) \text {, }
$$

where $X$ is an $n$-vector and $F(t, X)$ is an $n$-vector function defined on a region in $I \times \mathbb{R}^{n}$. The 0 -set of the set of points $(t, X)$ is denoted such that $t \in I, \dot{X}=0$.

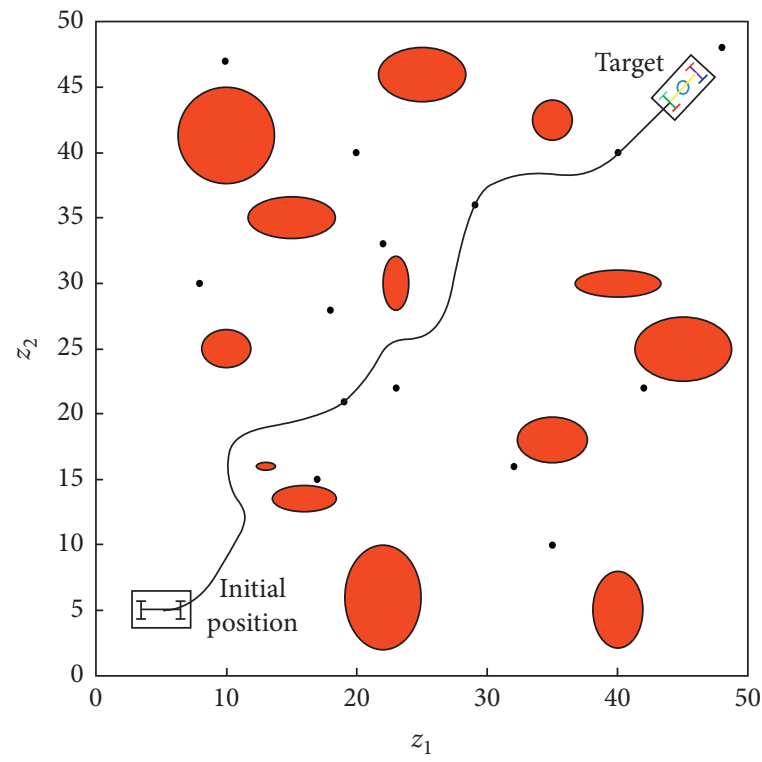

FIGURE 10: Landmark navigation of a car-like robot in an obstacleridden workspace with initial position at $(5,5)$ and target at $(45,45)$.

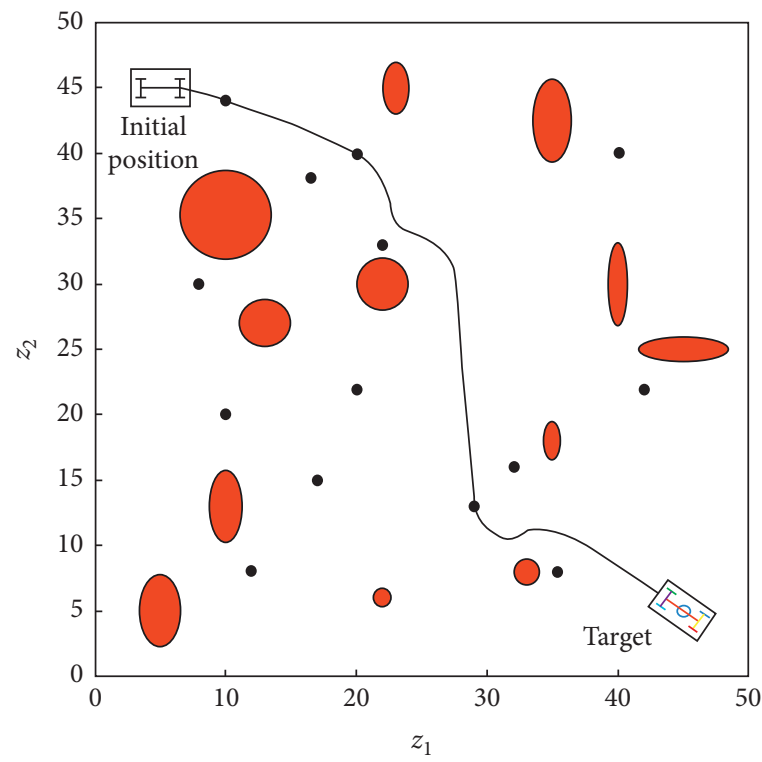

FIGURE 11: Landmark navigation of a car-like robot in an obstacleridden workspace with initial position at $(5,45)$ and target at $(45,5)$.

Theorem 2 (see Yoshizawa [49]). Suppose that $F(t, X)$ of (29) is continuous on $I \times S_{H}, H>0$, where $S_{H}$ denotes the set of $X$ such that $\|X\| \leq H$. Suppose that there exist a Lyapunov function $V(t, X)$ defined on $I,\|X\|<H$, which satisfies the following conditions:

(1) $a(\|X\|) \leq V(t, X) \leq b(\|X\|)$, where $a(r)$ and $b(r)$ are continuous increasing, positive definite functions.

(2) $\dot{V}_{(11)} \leq h(t) q(t, X)$, where $\int_{0}^{\infty}|h(t)| d t<\infty$ and $q(t, X)$ is bounded. 
TABLE 1: Values of the parameters used in the simulations.

\begin{tabular}{lc}
\hline \multicolumn{2}{c}{ Initial and final configuration } \\
$\begin{array}{lc}\text { Initial and final position } \\
\text { Initial orientation }\end{array}$ & $\begin{array}{c}\text { Refer to the figures } \\
\theta=0 \mathrm{rad}\end{array}$ \\
\hline \multicolumn{3}{c}{ Robot parameters } \\
$\begin{array}{l}\text { Dimensions } \\
\text { Safety parameters }\end{array}$ & $\begin{array}{c}\ell=3 \mathrm{~m}, w=1.4 \mathrm{~m} \\
\varepsilon_{1}=0.2 \mathrm{~m}, \varepsilon_{2}=0.1 \mathrm{~m}\end{array}$ \\
\hline \multicolumn{3}{c}{ Other parameters } \\
Workspace dimensions & $0 \leq z_{1} \leq 50,0 \leq z_{2} \leq 50$ \\
Obstacle avoidance & $d_{\max }=4$ \\
Field of view & $\zeta=\pi / 6$ \\
& $D^{*}=5$, \\
& $\mu=0.05$, \\
Constants & $\gamma=0.01$, \\
& $\phi_{\max }=7 \pi / 18 \mathrm{rad}$ \\
\hline
\end{tabular}

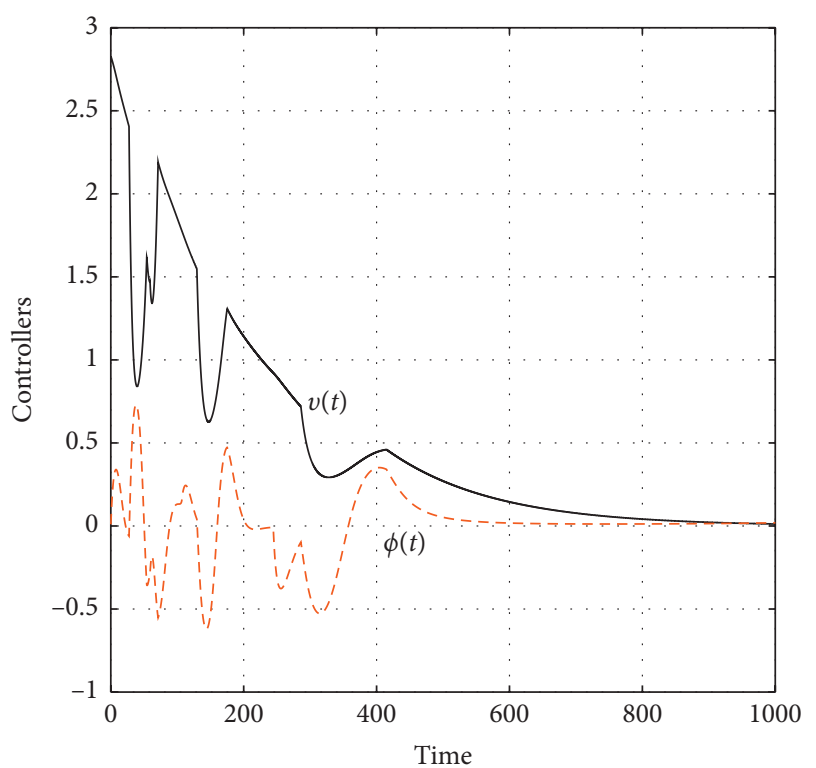

FIgURE 12: Evolution of controllers, $v(t)$ (solid line) and $\phi(t)$ (dotted line), for the trajectory shown in Figure 10.

Then the 0 -set is an eventually uniform stable set of system (29) with respect to $t$.

The next theorem shows how this concept of eventual stability is applied to system (9).

Theorem 3. The target $\mathbf{p}=\left(p_{1}, p_{2}\right)$ of system (22) is eventually uniform stable provided that the control inputs, $v$ and $\phi$, are as defined in (24).

Proof. The proof is adopted from [16]. Consider a Lyapunov function of the form

$$
L\left(t, \mathbf{x}^{*}(t)\right)=\frac{1}{2}\left\|\mathbf{x}^{*}(t)-\mathbf{p}\right\|^{2} e^{-t},
$$

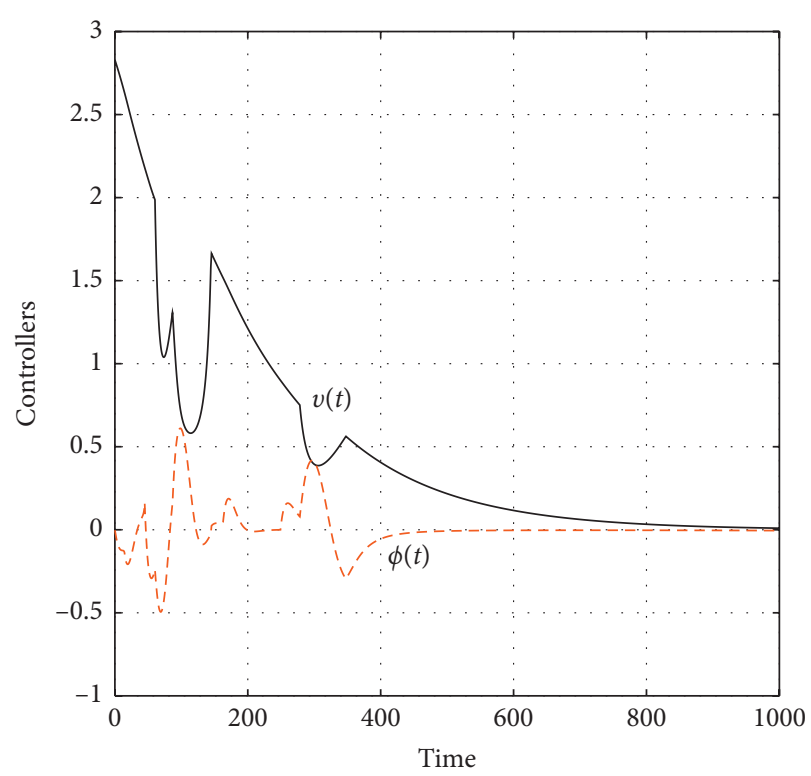

Figure 13: Evolution of controllers, $v(t)$ (solid line) and $\phi(t)$ (dotted line), for the trajectory shown in Figure 11.

defined on $I=[0, \infty)$ and $\left\|\mathbf{x}^{*}(t)-\mathbf{p}\right\|<H$ for some sufficiently large $H>0$. Clearly, our Lyapunov function satisfies the first condition of Theorem 2 in $\mathbb{R}^{2}$ since

$$
0 \leq L\left(t, \mathbf{x}^{*}(t)\right) \leq \frac{1}{2}\left\|\mathbf{x}^{*}(t)-\mathbf{p}\right\|^{2},
$$

and $\left\|\mathbf{x}^{*}(t)-\mathbf{p}\right\|^{2}$ is a continuously increasing positive definite function in $\mathbb{R}^{2}$. Next, along a planar trajectory of system (22), we see that

$$
\begin{aligned}
\dot{L}= & -\frac{1}{2}\left\|\mathbf{x}^{*}-\mathbf{p}\right\|^{2} e^{-t} \\
& +e^{-t} v\left[\left(x-p_{1}\right) \cos \theta(t)+\left(y-p_{2}\right) \sin \theta\right] \\
& -\frac{e^{-t} v \tan \phi}{2}\left[\left(x-p_{1}\right) \sin \theta-\left(y-p_{2}\right) \cos \theta\right] \\
= & -e^{-t}\left\{\frac{1}{2}\left(d^{*}\right)^{2}+v d^{*}\left[\cos (\theta-\psi)+\frac{1}{2} \tan \phi \sin (\theta-\psi)\right]\right\},
\end{aligned}
$$

where $d^{*}(t)=\left\|\mathbf{x}^{*}(t)-\mathbf{p}\right\|$ and $\psi(t)=\tan ^{-1}\left[\left(p_{2}-y(t)\right)\right.$ $\left./\left(p_{1}-x(t)\right)\right]$ for $\mathbf{x}^{*} \neq \mathbf{p}$. (Note that $\dot{L}(t, \mathbf{p})=0$ for all $t \geq 0$.) Now, since $\phi$ and $v$ are bounded as $|\phi| \leq \phi_{\max }$ and $v \leq v_{\max }$, it follows that

$$
\begin{aligned}
\dot{L} \leq & e^{-t}\left\{\frac{1}{2}\left\|\mathbf{x}^{*}(t)-\mathbf{p}\right\|^{2}\right. \\
& \left.+v_{\max }\left\|\mathbf{x}^{*}(t)-\mathbf{p}\right\|\left[1+\frac{1}{2} \tan \phi_{\max }\right]\right\} .
\end{aligned}
$$




$$
\begin{aligned}
& \text { Note that } \int_{0}^{\infty}\left|e^{-t}\right| \mathrm{d} t=1<\infty \text { and } \\
& \qquad \begin{aligned}
q\left(t, \mathbf{x}^{*}\right):= & \frac{1}{2}\left\|\mathbf{x}^{*}(t)-\mathbf{p}\right\|^{2} \\
& +v_{\max }\left\|\mathbf{x}^{*}(t)-\mathbf{p}\right\|\left[1+\frac{1}{2} \tan \phi_{\max }\right] \\
< & \frac{1}{2} H^{2}+v_{\max } H\left[1+\frac{1}{2} \tan \phi_{\max }\right],
\end{aligned}
\end{aligned}
$$

is bounded; thus, the second condition of Theorem 2 is also satisfied. Hence, the proof of Theorem 3 is complete.

\section{Discussion}

In this section, the strengths and weaknesses of the proposed method are discussed. Firstly, the algorithm proposed in this paper for landmark selection depends on the field of view and those points that lie in between the robot's position and its target. This ensures that all those points which lie behind the robot or behind the target are automatically discarded. For the landmark changeover, the differential form of the functions $\psi(t)$ ensures that the trajectories at all points (including the landmarks) are smooth. This means that the velocity controllers and the corresponding Lyapunov function are all continuous for all time $t \geq 0$. With a clear definition of the field of view, it is shown that all trajectories starting near the equilibrium point are guaranteed to converge to the equilibrium point. Hence, the equilibrium point is asymptomatically stable as seen in Theorem 1 .The technique and the new dynamic updating rule are applied to a car-like robot system. Through simulations, it is seen that the trajectory produced by the car-like robot is smooth at all points, and the graph of the control laws is continuous all the time. It was noted that the planar trajectory of the car-like system is, generally, eventually stable, which means that initially the system trajectory could move away from the target, for example, if the target is located on the sides or directly behind the robot, but after a certain time it converges to a vicinity of the target and remains there for all time $t \geq T$. A theorem proposed by Yoshizawa [24] in 1966 was used to carry out the stability analysis and theoretically show that the target point of our system is eventually uniform stable. On the other hand, this paper proposes only the velocity-based control laws. For $2 \mathrm{D}$ point robots, velocity controls are fine; however, for nonholonomic systems such as a car-like robot, it is important to accommodate dynamics of the system by including the acceleration. The accelerationbased control laws will allow motion control at higher speeds. The current kinematic model defined in (22) reduces the significance of the results to low speeds. This will be considered in the future.

\section{Conclusion}

The paper successfully provides a new stabilizing solution to the landmark navigation problem using a single autonomous robot. For the first time, the problem is solved using a time-variant, yet continuous, updating rule of the robot's steering angle and orientation parameters only, while the navigation to the target is facilitated by a newly constructed velocity-based algorithm.

The research becomes more interesting and challenging when multiple landmarks are introduced randomly in the workspace cluttered with randomly fixed obstacles. Note that different metrics have been designed in the literature for the selection of relevant landmarks required for the motion control problems. In this case, the authors have designed a new dynamic updating rule to select landmarks from a newly constructed metric to capture the field of view of the robot. The algorithm was then successfully applied to a car-like robot with nonholonomic constraints. The effectiveness of the proposed solution was consistently verified through computer simulations using Matlab and its stability proved theoretically.

While the concept of landmark navigation is recent, the potential for practical applications cut across a number of sectors and workplaces. One such workplace where the concept can be utilized is loading/offloading in a constrained environment such as docks. The landmarks can guide autonomous robots to park correctly in designated spots or parking bays for precise loading/offloading. Such artificial landmarks can also be used to find simpler vehicle routes in a network of streets and highways, especially during heavy traffic when the cost of taking different routes differs; hence, the landmarks can also assume different (negative/positive) roles. Similarly, for longer routes when the cost of having the entire journey predetermined and preloaded into autonomous vehicles is very high, again these landmarks can be very useful in providing the relevant guidance to far placed targets.

A limitation of this research paper is that the theoretical contribution is not proved with an experimental design using real robots. This provides scope for future undertakings. Future work will also consider landmark navigation problem of multiple three-dimensional robots with the inclusion of nonconvex obstacles and dynamic landmarks.

\section{Data Availability}

No data were used to support this study.

\section{Conflicts of Interest}

The authors declare that they have no conflicts of interest.

\section{References}

[1] S. Behere, "A generic framework for robot motor planning and control," Master's thesis, Royal Institute of Technology, Stockholm, Sweden, 2010.

[2] B. Sharma, J. Vanualailai, and S. Singh, "Motion planning and posture control of multiple n-link doubly nonholonomic manipulators," Robotica, vol. 35, no. 1-25, 2015.

[3] B. N. Sharma, J. Raj, and J. Vanualailai, "Navigation of carlike robots in an extended dynamic environment with swarm avoidance," International Journal of Robust and Nonlinear Control, vol. 28, no. 2, pp. 678-698, 2018. 
[4] L. Krick, M. E. Broucke, and B. A. Francis, "Stabilization of infinitesimally rigid formations of multi-robot networks," in Proceedings of the 47th IEEE Conference on Decision and Control, pp. 477-482, Cancun, Mexico, December 2008.

[5] I. Matychyn, "Pursuit strategy of motion camouflage in dynamic games," Dynamic Games and Applications, vol. 10, no. 1, pp. 145-156, 2020.

[6] A. Kilin, P. Bozek, Y. Karavaev, A. Klekovkin, and V. Shestakov, "Experimental investigations of a highly maneuverable mobile omniwheel robot," International Journal of Advanced Robotic Systems, vol. 14, no. 6, 2017.

[7] B. Sharma, J. Vanualailai, and A. Prasad, "Formation control of a swarm of mobile manipulators," Rocky Mountain Journal of Mathematics, vol. 41, no. 3, pp. 900-940, 2011.

[8] K. Shojaei, "Neural adaptive output feedback formation control of type $(m, s)$ wheeled mobile robots," International Journal of Adaptive Control and Signal Processing, vol. 29, pp. 855-876, 2015.

[9] K. Shojaei, "Neural adaptive output feedback formation control of type $(m, s)$ wheeled mobile robots," IET Control Theory \& Applications, vol. 11, no. 4, pp. 504-515, 2017.

[10] P. Bozek, M. Aiman Al Akkad, P. Blistan, and N. Ibrahim Ibrahim, "Navigation control and stability investigation of a mobile robot based on a hexacopter equipped with an integrated manipulator," International Journal of Advanced Robotic Systems, vol. 14, no. 6, 2017.

[11] A. Pandey, A. K. Kashyap, D. R. Parhi, and B. K. Patle, "Autonomous mobile robot navigation between static and dynamic obstacles using multiple anfis architecture," World Journal of Engineering, vol. 16, no. 2, pp. 275-286, 2019.

[12] X. Xing, R. Zhou, and L. Yang, "The current status of development of pedestrian autonomous navigation technology," in Proceedings of the 26th Saint Petersburg International Conference on Integrated Navigation Systems (ICINS), Saint Petersburg, Russia, May 2019.

[13] P. Boucher, "Waypoints guidance of differential-drive mobile robots with kinematic and precision constraints," Robotica, vol. 32, pp. 1-24, 2014.

[14] A. Prasad, B. Sharma, and J. Vanualailai, "A solution to the motion planning and control problem of a car-like robot via a single-layer perceptron," Robotica, vol. 32, no. 6, pp. 935-952, 2014.

[15] S. A. Kumar, J. Vanualailai, and B. Sharma, "Lyapunov functions for a planar swarm model with application to nonholonomic planar vehicles," in Proceedings of the 2015 IEEE Conference on Control Applications (CCA), pp. 19191924, Sydney, Australia, September 2015.

[16] A. Prasad, B. Sharma, J. Vanualailai, and S. A. Kumar, "A geometric approach to target convergence and obstacle avoidance of a nonstandard tractor-trailer robot," International Journal of Robust and Nonlinear Control, vol. 30, no. 13, pp. 4924-4943, 2020.

[17] M. Ataei and A. Yousefi-Koma, "Three-dimensional optimal path planning for waypoint guidance of an autonomous underwater vehicle," Robotics and Autonomous Systems, vol. 67, pp. 23-32, 2015.

[18] A. Devi, J. Vanualailai, S. A. Kumar, and B. Sharma, "A cohesive and well-spaced swarm with application to unmanned aerial vehicles," in Proceedings of the 2017 International Conference on Unmanned Aircraft Systems, pp. 698-705, Miami, FL, USA, June 2017.

[19] X. Xie, Y. Ding, and X. Huang, "A landmark detection and recognition algorithm for uav autonomous pitching," in Proceedings of the 2019 IEEE International Conference on
Unmanned Systems (ICUS), pp. 484-489, Beijing, China, October 2019.

[20] L. C. Chie and Y. W. Juin, "Artificial landmark-based indoor navigation system for an autonomous unmanned aerial vehicle," in Proceedings of the 2020 IEEE 7th International Conference on Industrial Engineering and Applications (ICIEA), pp. 756-760, Paris, France, January 2020.

[21] B. Wohlfender, "Studies on mechatronics: autonomous collaborative vehicles," 2010.

[22] A. Pandey, V. S. Panwar, M. E. Hasan, and D. R. Parhi, "V-REPbased navigation of automated wheeled robot between obstacles using PSO-tuned feedforward neural network," Journal of Computational Design and Engineering, vol. 7, no. 4, pp. 427-434, 2020.

[23] N. Gravish and V. L. George, "Robotics-inspired biology," Journal of Experimental Biology, vol. 221, no. 7, 2018.

[24] J. Ni, L. Wu, X. Fan, and S. X. Yang, "Bioinspired intelligent algorithm and its applications for mobile robot control: A survey," Computational Intelligence and Neuroscience, vol. 2016, Article ID 3810903, 2016.

[25] R. Pirník, M. Hruboš, D. Nemec, T. Mravec, and P. Božek, "Integration of inertial sensor data into control of the mobile platform," in Proceedings of the 2015 Federated Conference on Software Development and Object Technologies, J. Jan, J. Kostolny, and T. Gratkowski, Eds., Springer International Publishing, pp. 271-282, Cham, Switzerland, November 2017.

[26] X. Xi and E. H. Abed, "Formation control with virtual leaders and reduced communications," in Proceedings of the 44th IEEE Conference on Decision and Control, and the European Control Conference, pp. 1854-1860, Seville, Spain, December 2005.

[27] M. Collett, D. Harland, and T. S. Collett, "The use of landmarks and panoramic context in the performance of local vectors by navigating honeybees," The Journal of Experimental Biology, vol. 205, no. Pt 6, pp. 807-814, 2002.

[28] R. Möller, "Insects could exploit UV-green contrast for landmark navigation," Journal of Theoretical Biology, vol. 214, no. 4, pp. 619-631, 2002.

[29] J. Borenstein, H. R. Everett, L. Feng, and D. Wehe, "Mobile robot positioning: sensors and techniques," Invited Paper for the Journal of Robotic Systems, vol. 14, no. 4, pp. 231-249, 1997.

[30] E. Mair, M. Augustine, B. Jäger et al., “A biologically inspired navigation concept based on the landmark-tree map for efficient long-distance robot navigation," Advanced Robotics, vol. 28, no. 5, pp. 289-302, 2014.

[31] A. S. Etienne, R. Maurer, V. Boulens, A. Levy, and T. Rowe, "Resetting the path integrator: a basic condition for routebased navigation," Journal of Experimental Biology, vol. 207, pp. 1491-1508, 2004.

[32] R. A. Harris, P. Graham, and T. S. Collett, "Visual cues for the retrieval of landmark memories by navigating wood ants," Current Biology, vol. 17, no. 2, pp. 93-102, 2007.

[33] K. Ishii, A. Ishida, G. Saul, M. Inami, and T. Igarashi, “Active navigation landmarks for a service robot in a home environment," in Proceedings of the 5th ACM/IEEE International Conference on Human Robot Interaction, HRI 2010, pp. 99-100, Osaka, Japan, March 2010.

[34] E. Celaya, J. L. Albarral, P. Jimenez, and C. Torras, "Visuallyguided robot navigation: from artificial to natural landmarks," in Proceedings of the International Conference on Field and Service Robotics, pp. 287-296, Chamonix, France, July 2007.

[35] A. Dawadee, J. Chahl, and N. Nanda, "A method for autonomous navigation of uavs using landmarks," in 
Proceedings of the 16th Australian Aerospace Congress, Melbourne, Australia, February 2015.

[36] D. Pazderski, "Waypoint following for differentially driven wheeled robots with limited velocity perturbations," Journal of Intelligent \& Robotic Systems, vol. 85, no. 3-4, pp. 553-575, 2017.

[37] D. Busquets, C. Sierra, and R. L. de Màntaras, "A multiagent approach to qualitative landmark-based navigation," $A u$ tonomous Robots, vol. 15, no. 2, pp. 129-154, 2003.

[38] H. Hu and D. Gu, "Landmark-based navigation of mobile robots in manufacturing," in Proceedings of the 1999 7th IEEE International Conference on Emerging Technologies and Factory Automation Proceedings ETFA 99 Cat No. 99TH8467, pp. 121-128, Barcelona, Spain, October 1999.

[39] A. Bais, R. Sablatnig, and J. Gu, "Single landmark based selflocalization of mobile robots," in Proceedings of the $3 \mathrm{rd} \mathrm{Ca}$ nadian Conference on Computer and Robot Vision (CRV'06), Quebec, Canada, June 2006.

[40] H. Fujii, Y. Ando, Y. Ando, T. Yoshimi, and M. Mizukawa, "Shape recognition of metallic landmark and its application to self-position estimation for mobile robot," Journal of Robotics and Mechatronics, vol. 22, no. 6, pp. 718-725, 2010.

[41] H. Jagannathan, "Landmark-based robot navigation enhanced with color interest operators," in Proceedings of the SPIE, vol. 3959, pp. 480-489, San Diego, CA, USA, May 2000.

[42] S. Lee, "Use of infrared landmark zones for mobile robot localization," Industrial Robot: An International Journal, vol. 35, no. 2, pp. 153-159, 2008.

[43] A. Chand and S. Yuta, "Road-crossing landmarks detection by outdoor mobile robots," Journal of Robotics and Mechatronics, vol. 22, no. 6, pp. 708-717, 2010.

[44] L. Frommberger, Representing and Selecting Landmarks in Autonomous Learning of Robot Navigation, Vol. 5314, Springer, Berlin, Germany, 2008.

[45] X. Deng, E. Milios, and A. Mirzaian, "Landmark selection strategies for path execution," Robotics and Autonomous Systems, vol. 17, no. 17-3, 1996.

[46] S. Marsland, U. Nehmzow, and T. Duckett, "Learning to select distinctive landmarks for mobile robot navigation," Robotics and Autonomous Systems, vol. 37, no. 4, pp. 241-260, 2001.

[47] M. Beinhofer, J.. Müller, A. Krause, and W. Burgard, "Robust landmark selection for mobile robot navigation," in Proceedings of the IEEE International Conference on Intelligent Robots and Systems (IROS), Tokyo, Japan, November 2013.

[48] H. Lee, H. Choi, D. Jung, and S. Choi, "Deep neural networkbased landmark selection method for optical navigation on lunar highlands," IEEE Access, vol. 8, pp. 99010-99023, 2020.

[49] T. Yoshizawa, Stability Theory by Liapunov's Second Method, The Mathematical Society of Japan, Tokyo, Japan, 1966.

[50] A. Prasad, "Governing robotic motion via a single layer artificial neural network," Ph. D. thesis, University of the South Pacific, Suva, Fiji Islands, 2012.

[51] A. Prasad, B. Sharma, and J. Vanualailai, "A new stabilizing solution for motion planning and control of multiple robots," Robotica, vol. 34, no. 5, pp. 1071-1089, 2016. 